University of New Hampshire

University of New Hampshire Scholars' Repository

Space Science Center

Institute for the Study of Earth, Oceans, and

Space (EOS)

2000

\title{
COMPTEL 1.8 MeV all sky survey: The Cygnus region
}

S Pluschke

Max-Planck-Institut für extraterrestrische Physik

R Diehl

Max-Planck-Institut für extraterrestriche

V Schonfelder

Max-Planck-Institut für extraterrestrische Physik

G Weidenspointer

Max-Planck-Institut für extraterrestrische Physik

H Bloemen

Space Research Organization of the Netherlands

See next page for additional authors

Follow this and additional works at: https://scholars.unh.edu/ssc

Part of the Astrophysics and Astronomy Commons

\section{Recommended Citation}

COMPTEL 1.8 MeV all sky survey: The Cygnus region Plüschke, S. and Diehl, R. and Schönfelder, V. and Weidenspointner, G. and Bloemen, $\mathrm{H}$. and Hermsen, W. and McConnell, M. and Ryan, J. and Bennett, K. and Oberlack, U. and Knödlseder, J., AIP Conference Proceedings, 510, 35-39 (2000),

DOl:http://dx.doi.org/10.1063/1.1303169

This Conference Proceeding is brought to you for free and open access by the Institute for the Study of Earth, Oceans, and Space (EOS) at University of New Hampshire Scholars' Repository. It has been accepted for inclusion in Space Science Center by an authorized administrator of University of New Hampshire Scholars' Repository. For more information, please contact Scholarly.Communication@unh.edu. 


\section{Authors}

S Pluschke, R Diehl, V Schonfelder, G Weidenspointer, H Bloemen, W Hermsen, Mark L. McConnell, James M. Ryan, K Bennett, U Oberlack, and J Knodlseder 


\section{AIP $\mid$ Proceedings}

\section{COMPTEL $1.8 \mathrm{MeV}$ all sky survey: The Cygnus region}

S. Plüschke, R. Diehl, V. Schönfelder, G. Weidenspointner, H. Bloemen, W. Hermsen, M. McConnell, J. Ryan, K. Bennett, U. Oberlack, and J. Knödlseder

Citation: AIP Conference Proceedings 510, 35 (2000); doi: 10.1063/1.1303169

View online: http://dx.doi.org/10.1063/1.1303169

View Table of Contents: http://scitation.aip.org/content/aip/proceeding/aipcp/510?ver=pdfcov

Published by the AIP Publishing

Articles you may be interested in

All sky monitor for energetic $y$-rays

AIP Conf. Proc. 558, 594 (2001); 10.1063/1.1370831

The spectral variability of Cygnus $\mathrm{X}-1$ at MeV energies

AIP Conf. Proc. 510, 114 (2000); 10.1063/1.1303185

Gamma-ray line emission from OB associations and young open clusters

AIP Conf. Proc. 510, 40 (2000); 10.1063/1.1303170

COMPTEL all-sky imaging at $2.2 \mathrm{MeV}$

AIP Conf. Proc. 410, 1099 (1997); 10.1063/1.54175

The total cosmic diffuse gamma-ray spectrum from 9 to $30 \mathrm{MeV}$ measured with COMPTEL

AIP Conf. Proc. 410, 1218 (1997); 10.1063/1.54105 


\title{
COMPTEL 1.8 MeV All Sky Survey: The Cygnus Region
}

\author{
S.Plüschke*, R.Diehl*, V.Schönfelder*, G.Weidenspointner*, \\ H.Bloemen ${ }^{+}$, W.Hermsen ${ }^{+}$, M.McConnell ${ }^{\dagger}$, J.Ryan $^{\dagger}$, \\ K.Bennett ${ }^{@}$, U.Oberlack $^{* *}, J . K n o ̈ d l s e d e r{ }^{++}$ \\ *MPI f. extraterrestrische Physik, Postfach 1603, 85740 Garching, Germany \\ +SRON-Utrecht, Sorbonnelaan 2, NL-3584 CA Utrecht, The Netherlands \\ ${ }^{\dagger}$ Universtity of New Hampshire, Durham NH 03824-3525, USA \\ @ Astrophysics Division, ESTEC, NL-2200 AG Noordwijk, The Netherlands \\ ** Astrophysics Laboratories, Columbia University, New York, NY 10027, USA \\ ${ }^{++}$CESR, CNRS/UPS, 31028 Toulouse, France
}

\begin{abstract}
We present an updated version of COMPTEL's $1.809 \mathrm{MeV}$ sky survey. Based on eight years of observations we compare "esults from different imaging techniques using background from adjacent energy bands. We confirm the previously reported characteristics of the galactic $1.809 \mathrm{MeV}$ emission, specifically an extended galactic ridge emission, mainly concentrated towards the inner galaxy, a peculiar emission feature in the Cygnus region, and a low-intensity ridge extending towards Carina and Vela. Because this gamma ray line is due to the decay of radioactive ${ }^{26} \mathrm{Al}$, predominantly synthesized in massive stars, one anticipates flux enhancements aligned with regions of recent star formation. This is born out by the observations. In particular the Cygnus feature, first presented in 1996 based on three years of COMPTEL data, is confirmed. Based on the stellar population we distinguish three prominent areas in this region, for which we separately derive fluxes, and discuss interpretations.
\end{abstract}

\section{INTRODUCTION}

The imaging gamma-ray telescope COMPTEL [1] aboard the CGRO spacecraft allowed for the first time to survey the entire sky in the $\mathrm{MeV}$ regime. One of the mission highlights is the deduction of the first all-sky image in the $1.809 \mathrm{MeV}$ gamma-ray line, first detected by HEAO-C and SMM [2]. This emission line is attributed to the radioactive decay of ${ }^{26} \mathrm{Al}$ witil a lifetime of $1.0417 \mathrm{Myr}$. Based on three years of COMPTEL data Oberlack et al. [3] presented a first $1.809 \mathrm{MeV}$ all-sky image in 1996. The image reconstruction was based on a maximum entropy algorithm (ME) [4] using a background model based on adjacent energy bands for each observation period individually [3,5]. In 1997 Oberlack published a first update of the ${ }^{26} \mathrm{Al}$ all-sky map based on roughly five years of COMPTEL data in his $\mathrm{PhD}$ thesis [6]. This map confirmed the non-local character of the detected

CP510, The Fifth Compton Symposium, edited by M. L. McConnell and J. M. Ryan (C) 2000 American Institute of Physics 1-56396-932-7/00/\$17.00 
$1.809 \mathrm{MeV}$ emission, attributing most of the emission towards young massive stars and active star forming regions.

In addition to this work Knödlseder et al. 1999 [7] introduced a multi-resolution regularized expectation maximization (MREM) algorithm for image reconstruction. The MREM method uses a wavelet based noise reduction. This method suppresses artifacts by allowing only significant structures to appear in the image. Also with this method the prior reported emission characteristics are confirmed, although the MREM map is much less structured than the ME image. Note that the MREM approach seems somewhat conservative with respect to image structures whereas the maximum entropy images tend to show artifacts.

We followed both imaging approaches using all COMPTEL data up to end of mission phase 7 . In both cases we applied an improved version of the adjacent energy background model described below. Both methods reveal extended emission structures near Vela/Carina region as well as Cygnus as reported in the previous publications on the ${ }^{26} \mathrm{Al}$ all-sky maps.

In section 2 we give a short description of the data analysis. Section 3 discusses the two imaging approaches and the produced all-sky images, whereas section 4 presents the results of the detail analysis of the Cygnus region. In section 5 we summarize the results and discuss some interpretations.

\section{DATA ANALYSIS \& BACKGROUND MODELING}

Our present analysis includes all data from beginning of the mission up to the end of mission phase 7. This spans a total observation time from May 1991 to November 1998, split into more than 270 observations periods with typical durations of $10^{6} \mathrm{~s}$. For the imaging analysis the event data from a $200 \mathrm{keV}$ wide energy band around the line energy is binned in a 3-dimensional dataspace consisting of the scatter direction of the photon $(\chi, \psi)$ and the scatter angle $\bar{\varphi}$. For a more detailed description of the dataspace and the event selections see [3].

Due to the very low signal-to-noise ratio of roughly $1 \%$, background modeling is the crucial task in analyzing COMPTEL data. In the case of gamma-ray line analysis the use of adjacent energy bands as basis for a background model seems natural. This background model is applied on the basis of single observation periods as described earlier in [3] and references therem. In contrast to prior applications of this background model an advanced smoothing technique is applied, restoring the original event distribution characteristics. Furthermore this method garantuees an accurate treatment in the galactic polar regions.

A longterm study of count-ratios from the line band relative to a narrow adjacent band, used for background deduction, shows a clear time-dependence in the background normalization (see [6]). This time-variability was attributed to increased instrumental-line background. Details on COMPTEL's instrumental-line background are reported in this proceedings [8] and in [6]. Specifically the contributions from the radioactive decay of ${ }^{22} \mathrm{Na}$ and ${ }^{24} \mathrm{Na}$ contaminate the event distri- 
butions in the adjacent bands. To cancel the longterm trend in the background normalization the contributions from various instrumental background components are determined by spectral fits for each observation period separately. From the resulting decay rates a renormalisation coefficient is calculated. These coefficients are used to compute a summed background model for the observation periods under consideration.

\section{THE $1.809 \mathrm{MeV}$ MAPS}

We applied two imaging methods to construct all-sky images. First we used a ME algorithm (see [4]) which iteratively extracts sky intensity distributions being compatible with the data. The ME method shows a clear trend to create a lumpy, structured image in late iterations as Oberlack and Knödlseder [6,9] had shown. On the other hand early iterations significantly ur.derestimate the gamma-ray fluxes. For these reasons an intermediate iteration has been chosen as a compromise between flux reproduction and map smoothness (see left panel of figure 1).
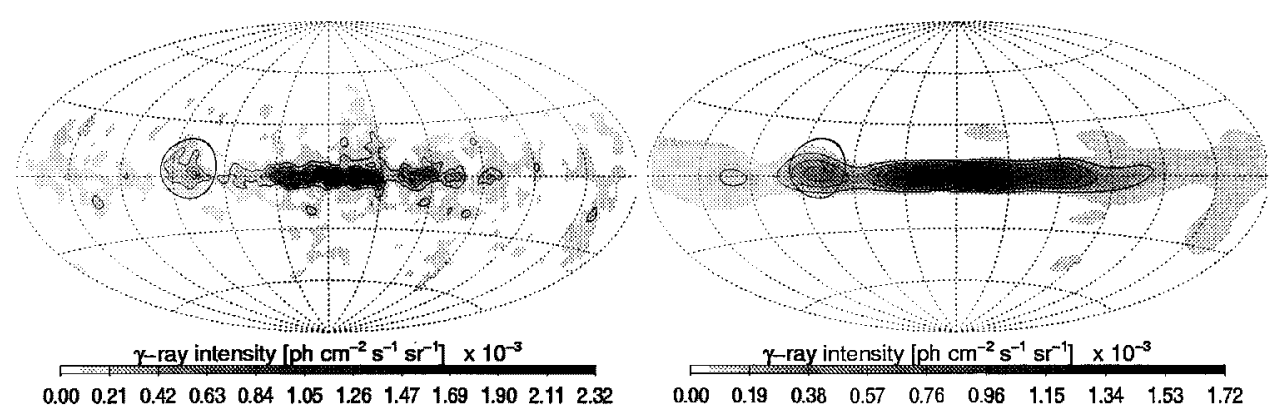

$\begin{array}{llllllllllll}0.00 & 0.21 & 0.42 & 0.63 & 0.84 & 1.05 & 1.26 & 1.47 & 1.69 & 1.90 & 2.11 & 2.32\end{array}$

FIGURE 1. 1.8 MeV Allsky Images (Cygnus Region encircled): Maximum Entropy Image (left) and the MREM Image (right)

As second approach we used the convergent MREM method [7] which combines an iterative Expectation Maximisation scheme with a wavelet analysis, filtering low significance structures and artifacts in the reconstruction. This method attempts to produce the smoothest map that is consistent with the data (see [7]). The MREM map is shown in figure 1 (right panel). The main features in both reconstructions are identical: an extended galactic ridge emission, mainly concentrated towards the inner galaxy, a peculiar emission feature towards Cygnus (see encircled region in the maps), and low-intensity ridge extending towards Carina and Vela. However, some low-level emission features towards the galactic anticentre visible in earlier maps disappeared, which might be due to the better statistics in our sample or the improved background treatment. Furthermore, low-level emission features appearing in the $\mathrm{ME}$ image between $180^{\circ}$ and $215^{\circ}$ longitude are suppressed in the MREM map. For the Cygnus analysis we furthermore applied a maximum likelihood (ML) imaging of this region (see figure 2). 


\section{$1.809 \mathrm{MeV}$ FROM THE CYGNUS REGION}

Based on the ME/ML images we analyzed the Cygnus region in detail following the analysis approaches presented in $[10,11]$. For a detailed description of this analysis see [12].

Figure 2 shows an overlay of our measurements with candidate point sources as well as the Cygnus OB associations. Five of these associations overlap in the Cygnus superbubble region (Cyg $\mathrm{X}$ region), which is also our strongest 1.8 $\mathrm{MeV}$ source region. We have determined fluxes for three regions ("Cygnus West" [Cyg X], "Cygnus East" [OB 7] and "Cygnus Arc" [arc-like structure north of the equator]), which are listed in table 1. As massive stars are the most promising ${ }^{26} \mathrm{Al}$ source candidates, WR stars and remnants from core-collapse $\mathrm{SNe}$ should explain the measured fluxes in the Cygnus region. Del Rio [10] found that WR stars in the regions could reproduce most of the observed flux. Our analysis reveals that up to $80 \%$ of the $1.8 \mathrm{MeV}$ flux could be directly attributed to the known isolated point sources [12]. This difference is due to the incorporation of more recent nucleosynthesis models and a different choice of the IMF; we use $\Gamma=1.35$ instead of $\Gamma=1.7$.



FIGURE 2. $1.8 \mathrm{MeV}$ ML Image of the Cygnus Region: Contours are significances whereas the grey-scale image shows the reconstructed source counts for each bin. The circles mark OB associations in the Cygnus Region whereas WR stars and SNRs are marked with dots and stars, respectively.

TABLE 1. measured $1.809 \mathrm{MeV}$ fluxes for Cygnus subregions; quoted errors are calculated via bootstrapping

\begin{tabular}{l|l||c|c|c}
\hline Region & Counterpart Region & $\begin{array}{c}\text { Longitude } \\
\text { deg }\end{array}$ & $\begin{array}{c}\text { Latitude } \\
\text { deg }\end{array}$ & $\begin{array}{c}\text { measured flux } \\
10^{-5} \mathrm{~cm}^{-2} \mathrm{~s}^{-1}\end{array}$ \\
\hline \hline Cygnus West & Cygnus Superbubble & $70-86$ & $-7-7$ & $3.7 \pm 1.1$ \\
\hline Cygnus East & Cygnus OB7 & $86-97$ & $-6-9$ & $2.0 \pm 0.6$ \\
\hline Cygnus Arc & & $76-94$ & $9-23$ & $2.0 \pm 0.6$ \\
\hline Cygnus Region & & $70-96$ & $-9-25$ & $7.9 \pm 2.4$ \\
\hline
\end{tabular}

Our model calculations of $\mathrm{OB}$ associations in the Cygnus region are still inconclusive due to large uncertainties in the normalisation of the initial mass function and star formation history. However, preliminary analysis indicates, that a large 
fraction of the observed fluxes can indeed be explained by the cumulative ${ }^{26} \mathrm{Al}$ yield from WR stars and core-collapse SN in these associations $[6,12]$.

\section{SUMMARY AND CONCLUSIONS}

In the previous sections we have shown the latest $1.809 \mathrm{MeV}$ maps deduced from COMPTEL data. As was shown in [13] the applied background model may suffer from misdeterminattion of continuum background from galactical sources. A detailed study of this problem is a still on-going task. Our maps confirm the reported structures aligned with areas of recent star formation. These sites are dominated by their massive star content. Specifically in the case of the Cygnus region we have determined fluxes and studied the possible source distribution. In the case of a static point source scenario we found that up to $80 \%$ of measured flux could be explained on the basis of recent nucleosynthesis calculations. The dynamic approach using a population synthesis model is somewhat inconclusive up to now due to large uncertainties in details of the population characterisation, but basically this model is also able to explain the measured $1.8 \mathrm{MeV}$ emission from Cygnus.

\section{REFERENCES}

1. Schönfelder V., et al., ApJS 86, 657-692 (1993)

2. Mahoney W.A., et al., ApJ 262, 742 (1982)

3. Oberlack U., et al., $A B A S 120,311-314$ (1996)

4. Strong A.W., et al., Data Analysis in Astronomy, V. Di Gesù et al. (eds.). Plenum Press New York, p. 251 (1992)

5. Knödlseder J., et al., SPIE Proc. Vol. 2806, Ramsey B.D., Parnell Th.A. (eds.), p. 386 (1996)

6. Oberlack U. PhD Thesis TU München (1997)

7. Knödlseder J., et al., $A \mathscr{B} A$ 345, 813-825 (1999)

8. Weidenspointner G., et al., in these Proceedings

9. Knödlseder J., PhD Thesis U Toulouse (1997)

10. del Rio E., et al., $A 63 A$ 315, 237 (1996)

11. Oberlack U., et al., Proc. of $2^{\text {nd }}$ Oak Ridge Symposium on Atomic and Nuclear Astrophysics, 2-6 Dec. 1997, p. 179 (1998)

12. Plüschke S., et al., these Proceedings

13. Bloemen H., et al. it Proc. Integral Workshop, Taormina (1998) 